\title{
Nerve transfers for elbow and finger extension reconstruction in midcervical spinal cord injuries
}

\author{
Jayme Augusto Bertelli, MD, PhD, and Marcos Flávio Ghizoni, MD, MSc \\ Department of Neurosurgery, University of the South of Santa Catarina (UNISUL), Tubarão, Brazil
}

\begin{abstract}
OBJECT The objective of this study was to report the results of elbow, thumb, and finger extension reconstruction via nerve transfer in midcervical spinal cord injuries.
\end{abstract}

METHODS Thirteen upper limbs from 7 patients with tetraplegia, with an average age of 26 years, were operated on an average of 7 months after a spinal cord injury. The posterior division of the axillary nerve was used to reinnervate the triceps long and upper medial head motor branches in 9 upper limbs. Both the posterior division and the branch to the middle deltoid were used in 2 upper limbs, and the anterior division of the axillary nerve in the final 2 limbs. For thumb and finger extension reconstruction, the nerve to the supinator was transferred to the posterior interosseous nerve.

RESULTS In 22 of the 27 recipient nerves, a peripheral type of palsy with muscle denervation was identified. At an average of 19 months follow-up, elbow strength scored M4 in 11 upper limbs and M3 in 2, according to the British Medical Research Council scale. Thumb extension scored M4 in 8 upper limbs and scored M3 in 4. Finger extension scored M4 in 12 hands. No donor-site deficits were reported or observed.

CONCLUSIONS Nerve transfers are effective at restoring elbow, thumb, and finger extension in patients with a midcervical spinal cord injury, which occurs in the majority of patients with a peripheral type of palsy with muscle denervation in their upper limbs. Efforts should be made to perform operations in these patients within 12 months of injury.

http://thejns.org/doi/abs/10.3171/2014.8.JNS14277

KEY WORDS axillary nerve; tetraplegia; elbow extension; nerve transfer; neurotization; peripheral nerve

$\mathrm{T}$ HE global incidence of spinal cord injury has been estimated to be between 10 and 80 per million individuals each year. The mean age of patients sustaining such injuries is 33 years old. One-third of these injuries results in tetraplegia. ${ }^{19}$ In a survey of patients with tetraplegia, $49 \%$ ranked reconstitution of upper-limb function as their first priority in rehabilitation, with no other priority surpassing $13 \% .{ }^{1}$ In the US, despite more than 100,000 citizens living with tetraplegia, fewer than 400 upper-extremity reconstructive procedures are performed each year. ${ }^{7}$

Most frequently, cervical spinal cord injury occurs at the C-6 level. This leaves the patient with preserved shoulder motion and elbow flexion, but elbow extension is absent. Despite preserved wrist extension, grasping is poor because thumb and finger flexion and extension are paralyzed (Group 2 in the International Classification system of muscle function in tetraplegia)..$^{12}$ Independence is sought by patients with tetraplegia, so an important goal of rehabilitation is to improve upper-limb function. Tendon transfer procedures restore basic upper-limb movements, improving abilities to groom, self-feed, self-catheterize, lift objects, write, swim, and drive. ${ }^{8}$ Reconstruction of elbow extension improves reaching capabilities and stabilizes the elbow, allowing for further reconstruction of grasping. ${ }^{10}$ However, using conventional methods, such as transferring either the posterior deltoid or biceps muscle to the triceps muscle, the vast majority of patients only recover enough strength to oppose gravity. ${ }^{10}$

Active thumb and finger extension is important for placing the fingers in adequate position for grasping, and fundamental for release. In patients with tetraplegia, no tendon procedure has demonstrated reliability and effectively ensured improved hand span. ${ }^{17}$

Nerve transfers consist of crossing one nerve to the other. Sacrificing the donor nerve does not lead to any loss of function, provided that the function of the nerve used can be compensated for via redundant muscle groups or innervation. The recipient nerve is the one responsible for an important missing function. Ideally, a nerve transfer

ABBREVIATION BMRC $=$ British Medical Research Council.

ACCOMPANYING EDITORIAL See pp 118-120. DOI: 10.3171/2014.5.JNS14964.

SUBMITTED February 11, 2014. ACCEPTED August 21, 2014.

INCLUDE WHEN CITING Published online October 24, 2014; DOI: 10.3171/2014.8.JNS14277.

DISCLOSURE The authors report no conflict of interest concerning the materials or methods used in this study or the findings specified in this paper. 
should involve synergistic nerves for which new function can be easily learned. In addition, these nerve transfers should involve motor branches transferred to recipient nerves very close to their muscle entry point to minimize delayed reinnervation and, consequently, enhance results. In patients with a mid-spinal cord lesion, the rationale is to transfer nerves with their motor neuron pool above the lesion to paralyzed nerves with a motor neuron pool below or at the lesion site.

For elbow extension reconstruction, transfer of axillary nerve branches to triceps motor branches has been proposed, while the branch of the supinator muscle has been transferred to reconstruct thumb and finger extension. ${ }^{3-6} \mathrm{In}$ spinal cord injuries, the use of nerve transfers is very new, and no clinical series have yet been published. In this paper we report our results on nerve transfers to reconstruct elbow, thumb, and finger extension in 13 upper limbs from 7 patients with tetraplegia.

\section{Methods \\ Study Patients}

From July 2011 to December 2012, a consecutive series of 6 male patients and 1 female patient, with a mean age of 26 years old, underwent surgical reconstruction an average of 7 months after their spinal cord injury. We included in our study only patients with complete spinal cord injury, at the C-6 spinal cord level, with less than 24 months of injury, and total paralysis of the lower limbs and perineal anesthesia. We excluded from the study patients without active wrist extension and those with incomplete tetraplegia. The local ethics committee approved this study, and all patients provided written consent prior to their participation.

In 13 upper limbs, shoulder motion, elbow flexion, and wrist extension were preserved and are the main subjects of this study. In 1 upper limb, shoulder motion was preserved, but elbow flexion and all function of the wrist and hand were absent. All patients had light touch perception preserved on the lateral aspect of the hand. Details of the patients and procedures are listed in Table 1.

\section{Surgical Procedures}

Under general anesthesia without muscle relaxants, both upper limbs underwent surgical reconstruction.

\section{Elbow Extension Reconstruction}

Through an axillary incision, the axillary nerve was dissected and electrically stimulated to check for functionality using an insulated 21-gauge needle (Contiplex D, B. Braun Melsungen AG) connected to a nerve stimulator (Stimuplex HNS 11, B. Braun Melsungen AG). The triceps long and upper medial head branches were identified, dissected proximally, and sectioned. In 9 upper limbs, the entire posterior division was sectioned as distally as possible and connected to the triceps branches. In 2 limbs, a branch to the middle deltoid together with the posterior division of the axillary nerve was used because only weak contractions were observed following posterior division stimulation. In the 2 remaining upper limbs, both in the same patient, electrical stimulation of the posterior division produced no muscle contraction, so the entire anterior division of the axillary nerve was used as a donor for transfer. All nerve coaptations were performed without any tension, with redundant length of the nerve stumps in such a way that motion of the upper limb, tested intraoperatively, did not disrupt the nerve coaptation. Nerve coaptation was secured using 9-0 sutures (Fig. 1). Details of the surgical technique can be reviewed elsewhere; 1 patient in this study was reported on previously in another paper. ${ }^{3}$

\section{Thumb and Finger Extension Reconstruction}

Through a posterior incision in the proximal third of the forearm, the posterior interosseous nerve was identified between the extensor carpi radialis brevis and the extensor digitorum communis after sectioning of the supinator muscle. The 2 branches to the supinator muscle arising from the posterior interosseous nerve were divided distally, while the posterior interosseous nerve was divided proximally but distal to the emergence of the supinator nerves. Supinator branches were then connected to the posterior interosseous nerve without tension using 9-0 sutures (Fig. 2). Details of the surgical technique can be obtained elsewhere. ${ }^{3,5}$ In 1 patient, an anterior elbow approach was used.

Before division for coaptation, all triceps branches and the posterior interosseous nerve were electrically stimulated. If muscle contraction was observed, palsy was deemed of central origin; if no muscle contraction was elicited, palsy was deemed of peripheral origin. The schematic representation of the nerve transfer procedure is depicted in Fig. 3.

\section{Postoperative Care}

Postoperatively, an above-elbow cast including the wrist was worn for 7 to 10 days. Caregivers were advised to avoid applying any pressure to the axillary region during patient transfers, as this could disrupt nerve coaptation.

Patients received no special physiotherapy training. They received 4 cycles of 5 doses of nandrolone, $50 \mathrm{mg}$ in the men and $25 \mathrm{mg}$ in the 1 woman, administered intramuscularly once every 15 days, with an interval of 30 days between cycles. During postoperative visits, patients were instructed that supination would produce thumb and finger extension. No particular training was administered to teach them that elbow extension was linked to deltoid activity.

\section{Associated Procedures}

After recovery of thumb and finger extension, at least 1 year after the initial surgery, distal radio-ulnar fusion was performed to avoid inadvertent supination during thumb and finger extension in 6 upper limbs. In 2 upper limbs, absence of proximal interphalangeal extension was addressed using House's ${ }^{11}$ procedure in the index and middle finger; in the ring and little finger, this problem was addressed by suturing the transverse lateral bands together over the extensor apparatus at the proximal interphalangeal joint, which produced centralization of the 


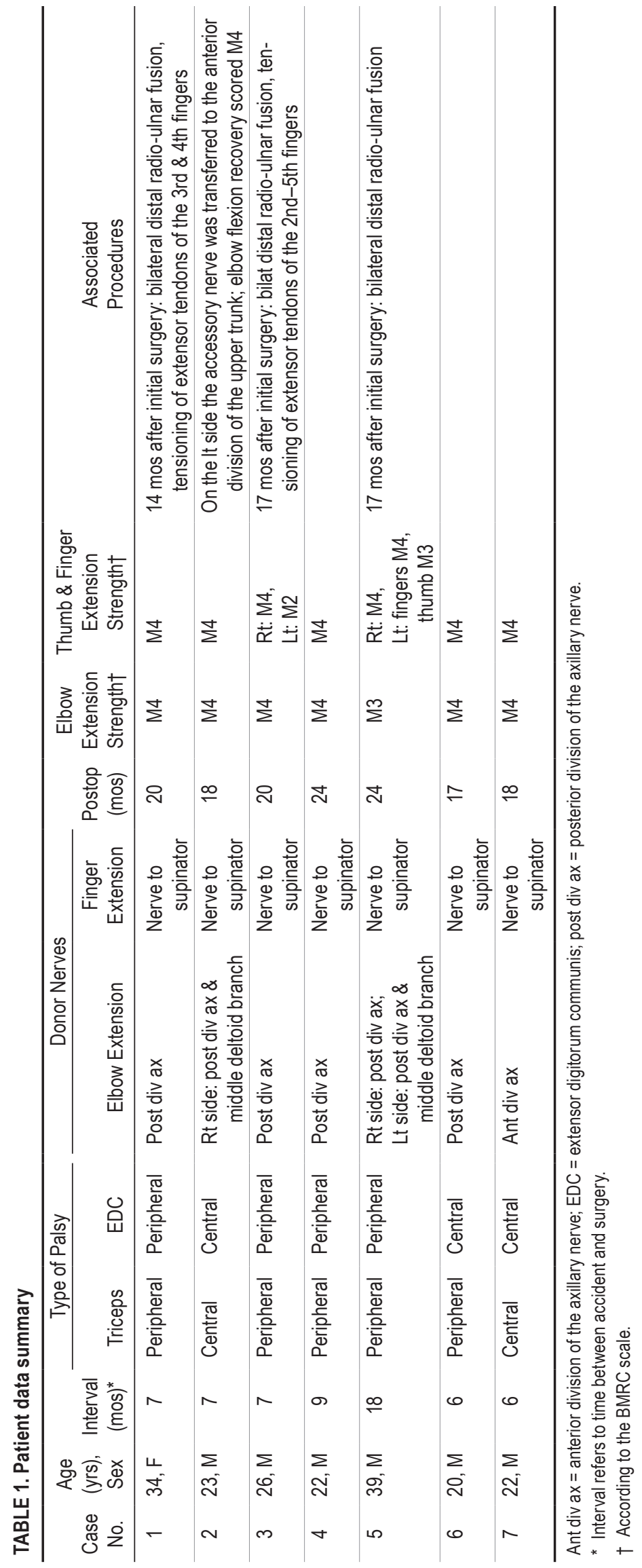




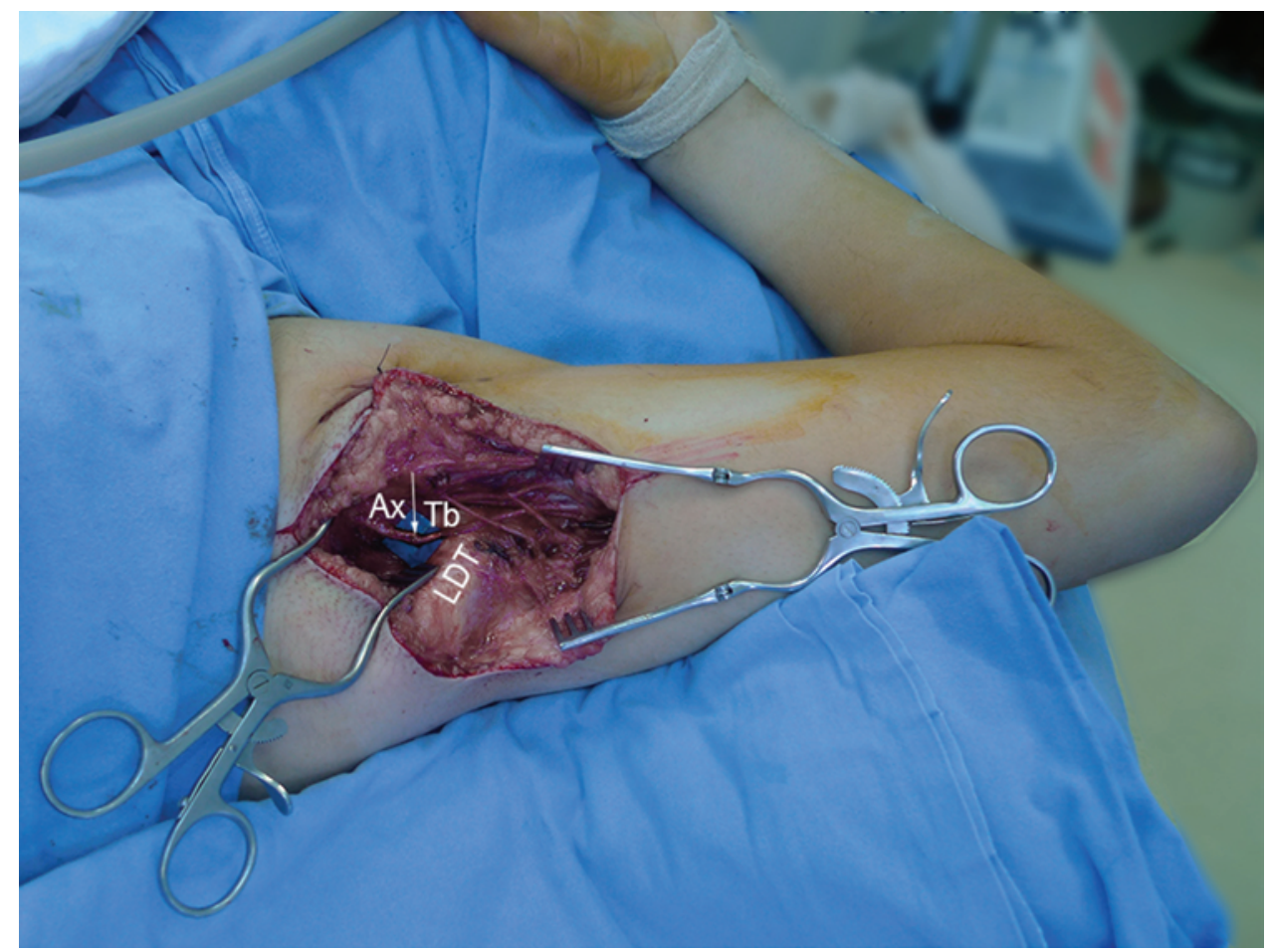

FIG. 1. Intraoperative view of the left axilla showing transfer of the posterior division of the axillary nerve (Ax) to triceps long and upper medial head motor branches $(\mathrm{Tb})$. The arrow indicates the site of nerve coaptation. $\mathrm{LDT}=$ latissimus dorsi tendon. Figure is available in color online only.

lateral bands of the extensor apparatus. House's procedure consists of using a tendon graft as a loop around the neck of the metacarpal, sutured on the extensor mechanism of 2 adjacent fingers. ${ }^{11}$

In 2 other upper limbs, only centralization of the lateral bands over the proximal interphalangeal joints of the middle and ring fingers was performed. We immobilized the proximal interphalangeal joints in extension for 3 weeks using K-wires.

\section{Postoperative Evaluation}

At the last visit, which occurred an average of 19 months after surgery, range of motion and strength of shoulder abduction, elbow extension, and thumb and finger extension were measured, with strength scored according to the British Medical Research Council (BMRC) guidelines. ${ }^{13}$ Supination was evaluated with the elbow flexed and extended. In those patients who ultimately underwent distal radio-ulnar fusion, this was conducted immediately before fusion surgery.

\section{Results}

Among the 27 recipient nerves, we identified a peripheral type of palsy in 22. At the time of the final postoperative assessment, elbow extension scored BMRC Grade M4 and was under full voluntary control in 11 upper limbs. In 2 upper limbs within the same patient, elbow extension scored Grade M3. All patients improved at selftransferring and controlling their wheelchairs.

Full thumb extension scoring M4 with the wrist in a neutral position was observed in 8 upper limbs. In 4 hands, thumb extension scored M3. In the last limb, active motion was observed but limited to half the full range (Grade M2). Full metacarpal extension scoring M4 was demonstrated in 12 hands, and this clearly improved hand span. Finger extension scoring M3 with only partial range of motion at the metacarpal phalangeal joint was observed in the remaining 1 limb.

Active extension at the proximal interphalangeal joint was observed in only 6 hands. Six patients learned to control unwanted supination during active thumb and finger extension, either by contracting their pronator teres muscle, which was weak but preserved in 4 patients, or by using the weight of the limb to counteract supination. After distal radio-ulnar fusion, all patients improved their hand span. Six patients intentionally limited their hand span prior to their fusion procedure to avoid unwanted supination. After surgery, all patients extended their thumb and fingers without restriction. None of the patients complained about losing forearm rotation.

After surgery, no decreased function was demonstrated at donor sites. No patient lost abduction strength or shoulder range (Table 2). Before distal radio-ulnar fusion, supination was preserved either with the elbow flexed or extended. Videos 1 and 2 show the results in 2 patients.

VIDEO 1. Clip showing pre- and postoperative views of Case 1. Copyright Jayme A. Bertelli. Published with permission. Click here to view with Media Player. Click here to view with Quicktime.

VIDEO 2. Clip showing pre- and postoperative views of Case 3. Copyright Jayme A. Bertelli. Published with permission. Click here to view with Media Player. Click here to view with Quicktime. 

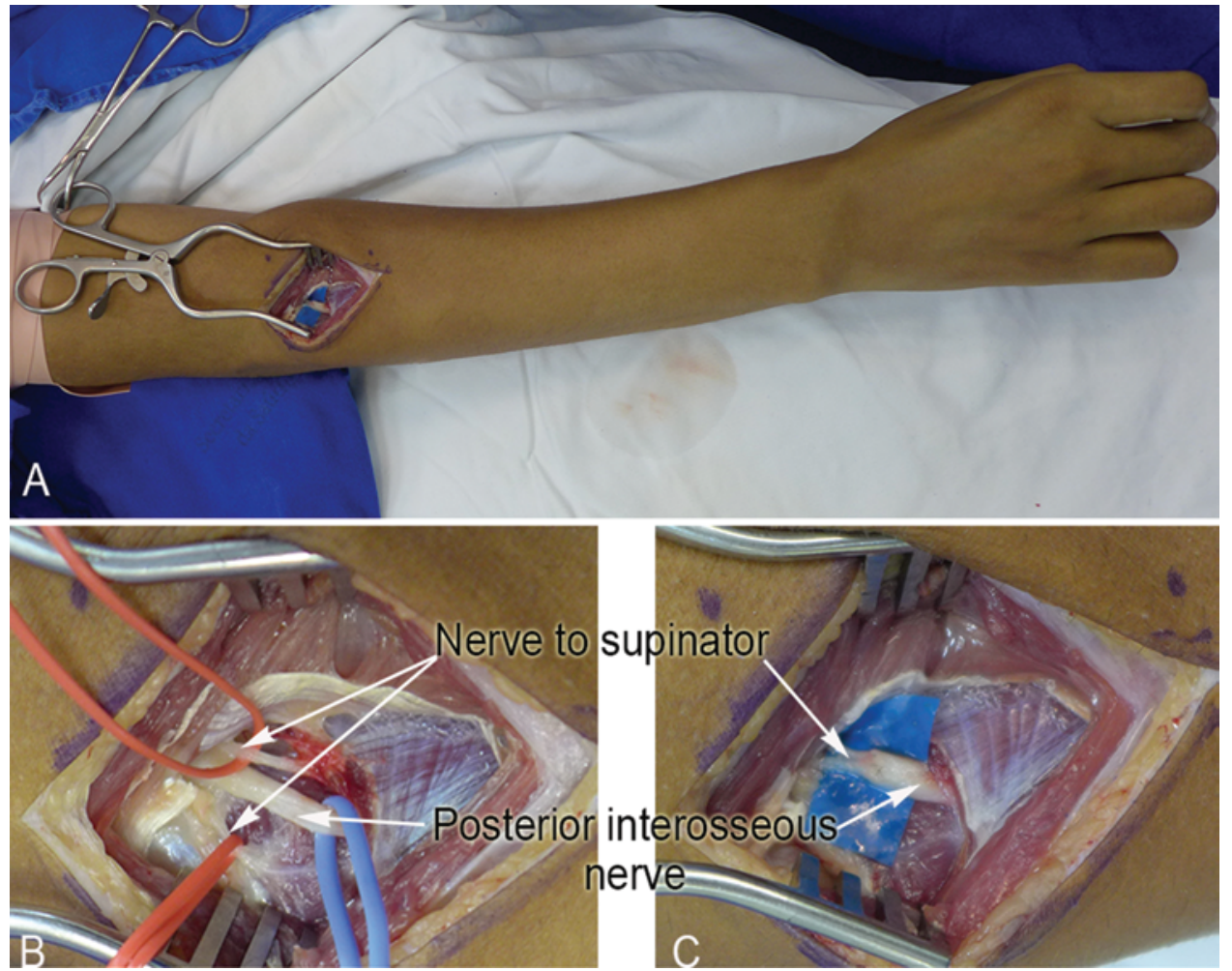

FIG. 2. Intraoperative view of the dorsal side of a right forearm showing transfer of the motor branches of the supinator muscle to the posterior interosseous nerve. A: Panoramic view. B: Dissection of the 2 motor branches of the supinator nerve and the posterior interosseous nerve before sectioning for transfer. C: Coaptation of the 2 motor branches of the supinator muscle with the posterior interosseous nerve. Figure is available in color online only.

\section{Discussion}

In our series of 13 upper limbs, elbow extension was predictably restored after axillary nerve branch transfer. All but 1 patient recovered Grade M4 strength. In the single case of Grade M3 recovery, the patient was almost 40 -years old, the oldest patient in our series, and underwent the operation 18 months after trauma for a peripheral type of palsy. A peripheral type of palsy was evident in 22 of the 27 recipient nerves. Peripheral palsy leads to Wallerian degeneration and muscle denervation with subsequent atrophy. Successful muscle reinnervation depends on the time between injury and repair. ${ }^{14}$ In spinal

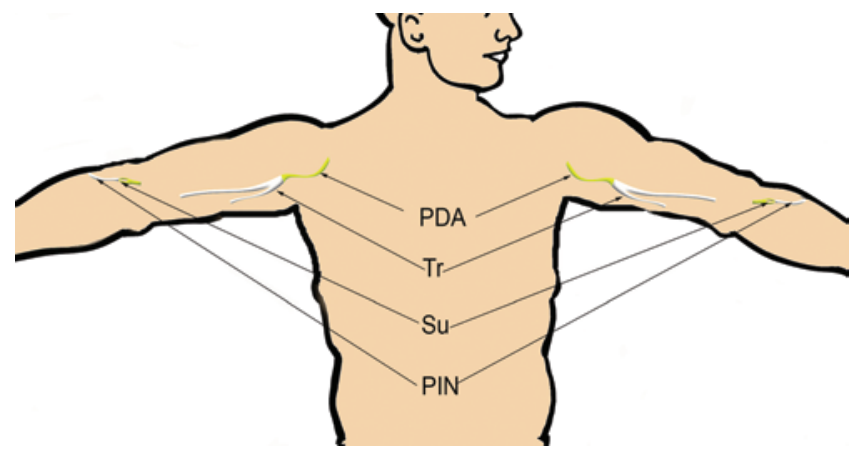

FIG. 3. Schematic representation of the transfer of the posterior division of the axillary nerve (PDA) to triceps motor branches (Tr). The nerve to the supinator muscle (Su) was transferred to the posterior interosseous nerve (PIN). Figure is available in color online only. cord trauma, peripheral palsy results from neuronal death at the site of the spinal cord injury. ${ }^{18}$ The high number of peripheral-type injuries we observed reinforces our initial belief that patients with tetraplegia should undergo operations within 1 year of their trauma. ${ }^{3-6}$ Of interest was the potential for improvement, even after 18 months of peripheral palsy, following a distal nerve transfer. We have deemed palsy to be of peripheral origin when intraoperative electrical stimulation of a given motor branch produced no muscle contraction. This was our gold standard because electrical stimulation under direct visual

TABLE 2. Pre- and postoperative range of motion and strength of shoulder abduction

\begin{tabular}{|c|c|c|c|c|c|c|c|c|}
\hline \multirow{3}{*}{$\begin{array}{l}\text { Case } \\
\text { No. }\end{array}$} & \multicolumn{4}{|c|}{$\begin{array}{c}\text { Shoulder Abduction Range } \\
\text { of Motion }\left({ }^{\circ}\right)\end{array}$} & \multicolumn{4}{|c|}{$\begin{array}{c}\text { Shoulder Abduction } \\
\text { Strength* }\end{array}$} \\
\hline & \multicolumn{2}{|c|}{ Preop } & \multicolumn{2}{|c|}{ Postop } & \multicolumn{2}{|c|}{ Preop } & \multicolumn{2}{|c|}{ Postop } \\
\hline & Rt & $\mathrm{Lt}$ & Rt & $\mathrm{Lt}$ & $\mathrm{Rt}$ & Lt & $\mathrm{Rt}$ & $\mathrm{Lt}$ \\
\hline 1 & 45 & 45 & 140 & 140 & M3 & M3 & M4 & M4 \\
\hline 2 & 140 & 0 & 160 & 0 & M4 & 0 & M4 & 0 \\
\hline 3 & 150 & 160 & 160 & 160 & M4 & M4 & M4 & M4 \\
\hline 4 & 140 & 140 & 160 & 160 & M4 & M4 & M4 & M4 \\
\hline 5 & 160 & 160 & 160 & 160 & M4 & M4 & M4 & M4 \\
\hline 6 & 100 & 100 & 140 & 140 & M3 & M3 & M4 & M4 \\
\hline 7 & 90 & 70 & 120 & 110 & M4 & M3 & M4 & M3 \\
\hline
\end{tabular}

* According to the BMRC scale. 
control eliminates the risks of sampling errors, which might occur following percutaneous electrical stimulation during routine electrophysiological examination. In this regard, our last 3 patients underwent electromyographic assessment of the triceps and extensor digitorum communis muscles, which demonstrated muscle denervation (not reported). We did not request preoperative electrophysiological examination in our first 4 cases because such a large extent of peripheral paralysis was unexpected. However, even in the situation of central paralysis, with preserved muscle trophism, we would recommend yearly surgery. At the moment, there is no clinical evidence that functional reinnervation of muscles following longstanding central paralysis is possible. In rats, it was demonstrated that muscles chronically paralyzed after long tract lesions of the spinal cord can be reinnervated, although strength recovery is limited to half of normal strength. ${ }^{18}$

In our first case report on a similar patient, we transferred only the teres minor nerve for triceps reinnervation. ${ }^{4}$ In the present series, in no instance did we identify strong contractions of the teres minor nerve upon nerve stimulation, so we proceeded to harvest the entire posterior division of the axillary nerve, which also innervates the posterior deltoid muscle. ${ }^{6}$ We had not attempted this before, because we were afraid of losing the posterior deltoid muscle as an option for transfer in case of failure. This is no longer of major concern because of the predictability of triceps muscle reinnervation by nerve transfer. Indeed, in cases of nerve transfer failure, the biceps muscle nerve can still be transferred to the triceps muscle, provided that the brachialis motor branch has not been used to reinnervate finger flexors. In such a situation, supination would be lost because the motor branch of the supinator would have been used to reinnervate the posterior interosseous nerve. However, as observed herein after distal radio-ulnar fusion, patients with tetraplegia at the C-6 level can cope without supination. In fact, they do not use forearm rotation because pronation is already impaired. In 2 upper limbs, we used the branch to the middle deltoid, and in 2 others we used the entire anterior division of the axillary nerve because the posterior division was paralyzed. Despite this method, patients experienced no deficits in shoulder function and did not complain of either weakness or fatigability. Possibly, the innervated suprascapular muscle compensated for the axillary nerve palsy. In fact, what we observed was improved shoulder abduction after harvesting axillary nerve branches for triceps muscle reinnervation. One possible explanation relates to augmented upper-limb use postoperatively. Preoperatively, if patients attempted abduction above horizontal, because of triceps paralysis, the forearm dropped down over their heads. This was corrected postoperatively, allowing patients to use their upper limb above horizontal. An interesting observation was that the anterior division of the axillary nerve, which innervates the anterior and middle deltoid muscles, ${ }^{6}$ was preserved in all patients, whereas posterior deltoid muscle innervation was impaired in 4 upper limbs. This suggests that the anterior deltoid muscle receives substantial innervation from the C-5 spinal cord level, which was not damaged in our patients. On the other hand, peripheral paralysis of the posterior division of the axillary nerve would be associated with damage to motor neurons at the C-6 spinal cord level, which would contribute substantially to posterior deltoid innervation.

Among the 13 upper limbs, thumb and finger extension were restored in 12 . We had only 1 case we considered a failure because of partial recovery, which could have been caused by a poor donor nerve or a technical problem during surgery. In this patient, we dissected the supinator nerve via an anterior incision over the elbow, because we used the brachialis tendon prolonged with a tendon graft to reconstruct finger flexion. We initiated immediate active finger flexion to avoid tendon adhesions with surrounding tissues, which would preclude tendon graft gliding and ultimately finger motion. Hence, because of the proximity of our tendon graft with the supinator to the posterior interosseous nerve transfer, we cannot exclude partial disruption of the coaptation site. Possibly a posterior arm approach, as performed in the remaining cases, would had been safer. Of particular importance was restoration of thumb motion and stability. None of our patients required any stabilization procedure such as thumb carpometacarpal fusion. We believe that the improved thumb stabilization our patients experienced resulted from reinnervation of their extensor pollicis brevis and abductor pollicis longus muscles. In general, patients with tetraplegia allow gravity to flex their wrist, extending their fingers via a tenodesis effect. ${ }^{14}$ However, in this position grasping is weak and awkward. Our patients could extend their thumb and fingers with their wrist either in the neutral position or extended, which improves grasping. The supinator nerve shares its level of innervation with wrist extensors. ${ }^{6}$ Hence, the stronger wrist extension is, the better supinator nerve quality is. However, we observed that even patients with weak wrist extension (i.e., Grade M3) can benefit from supinator nerve transfer. The initiator of thumb and finger extension motion was supination. In 6 patients, strong thumb and finger extension produced supination because of biceps muscle activation and the absence of active pronators. When we manually stabilized forearm rotation, we observed improved thumb and finger extension. In those patients in whom we fused the distal radio-ulnar joint, improved finger motion resulted in no noticeable deficits or complaints from patients about losing forearm rotation.

We used small doses of nandrolone during the postoperative period. Nandrolone decanoate is an anabolic steroid, which in a rat model of deficient reinnervation was found to accelerate nerve regeneration by $40 \% .{ }^{8}{ }^{9}$ Nandrolone in low doses is safe and has few adverse effects. ${ }^{15}$ Anabolic steroids can be bought with a prescription in several countries, including the US, ${ }^{15}$ although nandrolone has received FDA approval only for the treatment of renal insufficiency anemia. ${ }^{16}$ However, its use in clinical conditions associated with muscle wasting has increased markedly in recent years in the US. ${ }^{2}$

We did not report our results for finger flexion reconstruction, because of the diversity of procedures we used (brachialis tendon and nerve transfer, and brachioradialis tendon transfer) and the limited number of patients, which would preclude any definitive conclusions. 


\section{Conclusions}

In this study we describe how we performed initial nerve surgery bilaterally in a single stage with a short immobilization period. This procedure represents a major advantage relative to previous tendon transfer procedures, which required multiple-stage surgery and prolonged periods of immobilization. ${ }^{12}$ As such, we believe that nerve transfers are a reliable alternative for elbow, thumb, and finger extension reconstruction in patients with tetraplegia, and should be performed within 12 months of injury, provided that the large majority of paralysis is associated with muscle denervation.

\section{References}

1. Anderson KD: Targeting recovery: priorities of the spinal cord-injured population. J Neurotrauma 21:1371-1383, 2004

2. Basaria S, Wahlstrom JT, Dobs AS: Clinical review 138: Anabolic-androgenic steroid therapy in the treatment of chronic diseases. J Clin Endocrinol Metab 86:5108-5117, 2001

3. Bertelli JA, Ghizoni MF: Single-stage surgery combining nerve and tendon transfers for bilateral upper limb reconstruction in a tetraplegic patient: case report. J Hand Surg Am 38:1366-1369, 2013

4. Bertelli JA, Ghizoni MF, Tacca CP: Transfer of the teres minor motor branch for triceps reinnervation in tetraplegia. Case report. J Neurosurg 114:1457-1460, 2011

5. Bertelli JA, Tacca CP, Ghizoni MF, Kechele PR, Santos MA: Transfer of supinator motor branches to the posterior interosseous nerve to reconstruct thumb and finger extension in tetraplegia: case report. J Hand Surg Am 35:1647-1651, 2010

6. Bertelli JA, Tacca CP, Winkelmann Duarte EC, Ghizoni MF, Duarte H: Transfer of axillary nerve branches to reconstruct elbow extension in tetraplegics: a laboratory investigation of surgical feasibility. Microsurgery 31:376-381, 2011

7. Curtin CM, Gater DR, Chung KC: Upper extremity reconstruction in the tetraplegic population, a national epidemiologic study. J Hand Surg Am 30:94-99, 2005

8. Curtin CM, Hayward RA, Kim HM, Gater DR, Chung KC: Physician perceptions of upper extremity reconstruction for the person with tetraplegia. J Hand Surg Am 30:87-93, 2005

9. Ghizoni MF, Bertelli JA, Grala CG, da Silva RM: The anabolic steroid nandrolone enhances motor and sensory functional recovery in rat median nerve repair with long interpositional nerve grafts. Neurorehabil Neural Repair 27:269-276, 2013

10. Hamou C, Shah NR, DiPonio L, Curtin CM: Pinch and elbow extension restoration in people with tetraplegia: a systematic review of the literature. J Hand Surg Am 34:692699, 2009

11. House JH, Shannon MA: Restoration of strong grasp and lateral pinch in tetraplegia: a comparison of two methods of thumb control in each patient. J Hand Surg Am 10:22-29, 1985

12. Hentz VR, LeClercq C: Surgical Rehabilitation of the Upper Limb in Tetraplegia. New York: Saunders, 2002

13. James MA: Use of the Medical Research Council muscle strength grading system in the upper extremity. J Hand Surg Am 32:154-156, 2007

14. Jonsson S, Wiberg R, McGrath AM, Novikov LN, Wiberg M, Novikova LN, et al: Effect of delayed peripheral nerve repair on nerve regeneration, Schwann cell function and target muscle recovery. PLoS ONE 8:e56484, 2013

15. Kicman AT: Pharmacology of anabolic steroids. Br J Pharmacol 154:502-521, 2008

16. Kuhn CM: Anabolic steroids. Recent Prog Horm Res 57:411-434, 2002

17. Lamb DW, Landry R: The hand in quadriplegia. Hand 3:3137, 1971

18. Master D, Cowan T, Narayan S, Kirsch R, Hoyen H: Involuntary, electrically excitable nerve transfer for denervation: results from an animal model. J Hand Surg Am 34:479-487, e1-e3, 2009

19. Wyndaele M, Wyndaele JJ: Incidence, prevalence and epidemiology of spinal cord injury: what learns a worldwide literature survey? Spinal Cord 44:523-529, 2006

\section{Author Contributions}

Conception and design: Bertelli. Acquisition of data: both authors. Analysis and interpretation of data: Bertelli. Drafting the article: both authors. Critically revising the article: Bertelli. Reviewed submitted version of manuscript: Bertelli. Approved the final version of the manuscript on behalf of both authors: Bertelli.

\section{Supplemental Information \\ Videos}

Video 1, Media Player. http://mfile.akamai.com/21490/wmv/ digitalwbc.download.akamai.com/21492/wm.digitalsource-naregional/jns14-277_video_1.asx.

Video 1, Quicktime. http://mfile.akamai.com/21488/mov/ digitalwbc.download.akamai.com/21492/qt.digitalsource-global/ jns14-277_video_1.mov.

Video 2, Media Player. http://mfile.akamai.com/21490/wmv/ digitalwbc.download.akamai.com/21492/wm.digitalsource-naregional/jns14-277_video_2.asx.

Video 2, Quicktime. http://mfile.akamai.com/21488/mov/ digitalwbc.download.akamai.com/21492/qt.digitalsource-global/ jns14-277_video_2.mov.

\section{Correspondence}

Jayme A. Bertelli, Rua Newton Ramos 70, apto 901, Florianópolis, Santa Catarina, 88015395, Brazil. email: drbertelli@gmail.com. 